Tristan Lee, MD

Columbia University Irving Medica

Center, Department of Internal Medicine,

New York, NY
Jeffrey M. Clarke, MD

Duke Cancer Institute,

Durham, NC
Deepali Jain, MD

Department of Pathology, All India Institute

of Medical Sciences, Delhi, India
Sendhilnathan Ramalingam, MD

Duke Cancer Institute, Durham, NC
Vishal Vashistha, MD

Raymond G. Murphy New Mexico Veterans Affairs

Medical Center, Section of Hematology/Oncology,

Albuquerque, NM

\title{
Precision treatment for metastatic non-small cell lung cancer: A conceptual overview
}

\section{ABSTRACT}

Recent developments in precision oncology have increased the complexity of diagnostic and therapeutic decisions. Here, we broadly review the field of precision oncology and discuss common mutational drivers in non-small cell lung cancer (NSCLC) that directly relate to the diagnosis, evaluation, and treatment of patients with metastatic disease.

\section{KEY POINTS}

A number of driver alterations (mutations and chromosomal rearrangements) occur in patients with NSCLC.

Mutations in the EGFR and BRAF genes and rearrangements involving the $A L K$ and $R O S 1$ genes can be targeted with novel agents.

These targeted therapies have demonstrated superior outcomes and far less toxicity compared with traditional cytotoxic chemotherapy in patients with metastatic NSCLC.

Efficiently identifying genetic alterations that can be treated with existing therapies is key to providing bestpractice care to all patients.
T THE PAST FEW YEARS, targeted therapies have become widely available and have revolutionized the treatment of patients with advanced solid tumors, particularly metastatic non-small cell lung cancer (NSCLC). For patients who have 1 of a select few actionable genetic alterations, phase 3 trials in NSCLC have consistently shown survival benefits associated with targeted agents compared with chemotherapy. ${ }^{1-3}$ Large-scale real-world data suggest these targeted therapies are improving survival on a population level. ${ }^{4}$

Targeted therapies are costly, with estimates of cost per quality-adjusted life-year of $\$ 150,000$ to over $\$ 200,000$. However, they are also associated with improved quality of life and fewer adverse effects compared with chemotherapy. ${ }^{3,5-8}$

The drugs fall under the expanding umbrella term of "precision oncology," which refers to both the diagnostic method (ie, genomic sequencing) and the treatments prescribed based on the results. Recent advances in genomic sequencing have allowed for efficient and reliable identification of patients who may benefit from precision therapies.

Here, we review precision oncology and the most clinically relevant mutations that can be found among patients with metastatic NSCLC. We further review the diagnostic tests available to clinicians to assess for these mutations. Last, we discuss opportunities to streamline testing in an efficient manner. 


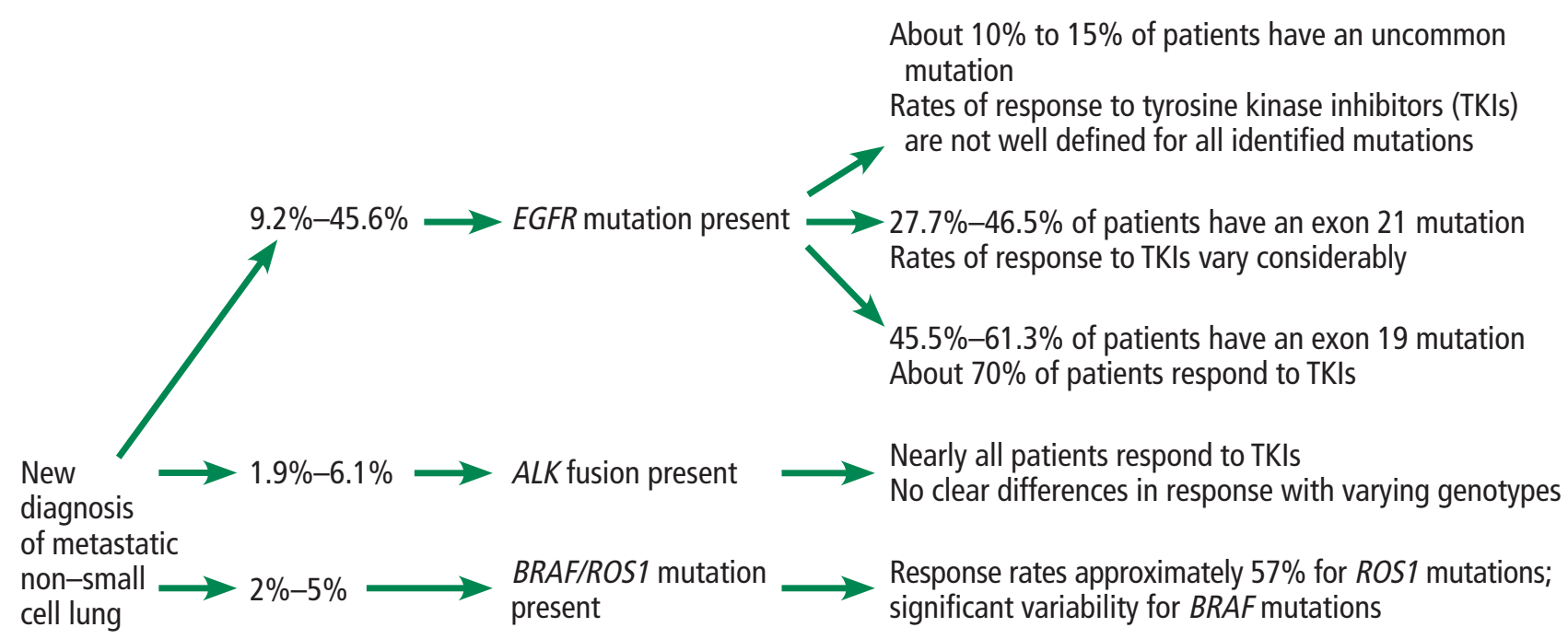

About $10 \%$ to $15 \%$ of patients have an uncommon mutation

Rates of response to tyrosine kinase inhibitors (TKIs) are not well defined for all identified mutations

cancer

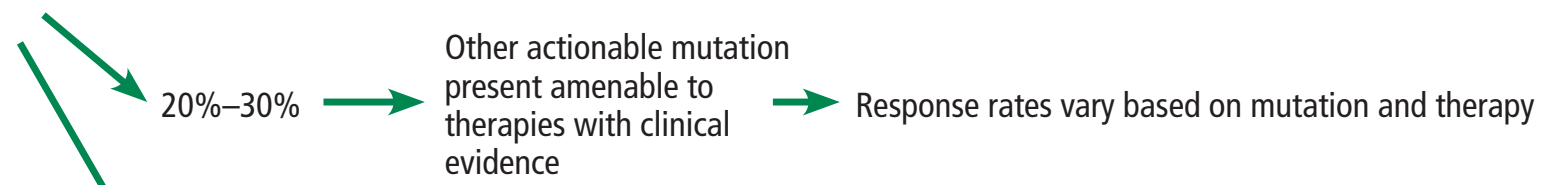

Frontline options include:
Cisplatin + paclitaxel
Carboplatin + paclitaxel

$\begin{array}{ll} & \text { Frontline options include: } \\ & \text { Cisplatin + paclitaxel } \\ & \text { Carboplatin + paclitaxel }\end{array}$

$40 \%-50 \% \longrightarrow \begin{aligned} & \text { No actionable mutation } \\ & \text { present }\end{aligned} \rightarrow \begin{aligned} & \text { Gemcitabine + cisplatin } \\ & \text { Cisplatin + pemetrexed }\end{aligned}$

Pembrolizumab based on PD-L1 status

Pembrolizumab + chemotherapy

Figure 1. The current paradigm for precision oncology for NSCLC.

\section{PRECISION ONCOLOGY}

Advances in the diagnosis and treatment of NSCLC have come to define the paradigm of precision oncology (Figure 1). Through remarkable laboratory-based efforts and wideranging epidemiologic studies, a significant number of critical genetic alterations that cause cells to grow, divide, and turn cancerous have been discovered.

As opposed to other accompanying and functionally neutral ("passenger") mutations, these specific "driver" mutations are functionally important to the growth of the malignancy. ${ }^{9}$ Further investigation into these driver mutations uncovered targeted therapies that provide a line of highly efficacious treatments, significantly improving overall survival for patients with metastatic NSCLC.

These developments have fundamentally altered clinicians' approaches to intervention in NSCLC over the past decade. Additionally, successes achieved in patients with NSCLC have encouraged further research efforts toward expanding the role of precision oncology for patients with other advanced malignancies.

In this review, we do not discuss immunotherapy, which is a general term referring to immune checkpoint inhibitors, namely agents that alter the cytotoxic T-lymphocyteassociated protein 4 and programmed deathligand 1 pathways. These agents have also vastly reshaped the treatment paradigm for patients with metastatic NSCLC, but specifically have a far greater role in patients who do not have a highly actionable mutation or fusion. The topic of immunotherapy is part of a broader discussion than is possible in this review. 


\section{GENETIC ALTERATIONS FOR WHICH THERAPIES ARE APPROVED}

Several genetic alterations identified in patients with metastatic NSCLC can currently be targeted with therapies approved by the US Food and Drug Administration (FDA), including mutations in the epidermal growth factor receptor (EGFR) and BRAF genes and chromosomal rearrangements of the anaplastic lymphoma kinase $(A L K)$ and ROS1 genes. The rates of alterations are shown in Figures 1 and 2. The associated targeted therapies for the different alterations are described in Table $1^{10-22}$ and Figure 2. Definitions and examples of key terms used in this article are given in Table 2, while a schematic review of the consequences of various actionable alterations is shown in Figure 3.

\section{EGFR mutations}

EGFR is a transmembrane tyrosine kinase receptor that operates within signal transduction pathways facilitating cellular growth and apoptosis. In the United States, nearly 20\% of patients with NSCLC harbor a pathogenic EGFR mutation. ${ }^{23}$ Mutations in the EGFR gene, which codes for the EGFR receptor, lead to dimerization of receptors. This dimerization causes constitutive activity of the tyrosine kinase associated with the EGFR protein, thereby inducing a hyperproliferative state.

Targeted treatments are directed toward inhibiting either the extracellular receptor or the intracellular tyrosine kinase. Among patients with metastatic NSCLC, efforts to inhibit intracellular tyrosine kinase have been most successful. The following drugs that inhibit EGFR tyrosine kinase are FDA-approved:

- Erlotinib, a first-generation drug

- Gefitinib, a first-generation drug

- Afatinib, a second-generation drug

- Dacomitinib, a second-generation drug

- Osimertinib, a third-generation drug.

A number of mutations can be found within the EGFR gene. The variants that are most susceptible to targeted treatments include exon 19 deletions and exon 21 substitutions (L858R). Cancers associated with less common mutations involving exon 18 and 20 may respond to tyrosine kinase inhibitor (TKI)based therapy, but sensitivity varies by specific

\section{Actionable alterations among patients with non-small cell lung cancer}

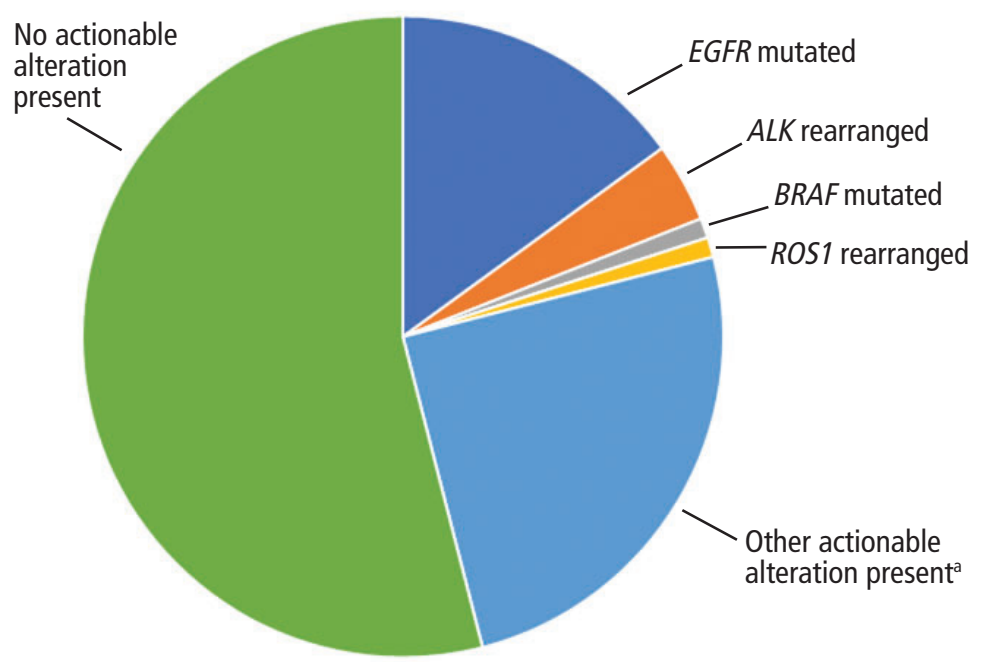

Figure 2. Rates of actionable mutations in patients with non-small cell lung cancer (NSCLC). Of note, NSCLC encompasses about $85 \%$ of lung cancers. Compared with smokers, nonsmokers have far higher rates of actionable mutations.

aThough another $20 \%$ to $30 \%$ of patients with NSCLC have some form of actionable alteration, the corresponding targeted agents are not necessarily FDA-approved. Of note, drugs targeting MET and RET have recently been approved for suitable NSCLC candidates.

mutation and is often lower compared with exon 19 and 21 mutations.

A number of clinical trials have demonstrated marked improvements in overall survival with use of TKIs compared with traditional chemotherapy in patients with an EGFR mutation. Later-generation TKIs such as osimertinib not only overcome a common mechanism of resistance, the T790M mutation, but also provide better progression-free and overall survival outcomes than earliergeneration TKIs for all patients with metastatic NSCLC harboring typical pathogenic EGFR mutations. ${ }^{24}$

Common adverse effects with TKIs are predominantly cutaneous, namely acneiform rash and dry skin, followed by diarrhea. Rarely, patients may develop interstitial lung disease. This is not an exhaustive list of potential adverse effects and neither are the adverse effect profiles described for the targeted therapies listed for patients harboring actionable alterations in ALK, ROS1, or BRAF. 


\section{TABLE 1}

\section{Approved targeted therapies for non-small cell lung cancer and their comparative effectiveness}

\section{Phase 3 trials consistently show marked survival benefits with targeted agents}

\begin{tabular}{|c|c|c|c|}
\hline Target & Treatment & Mechanism & $\begin{array}{l}\text { Median progression- } \\
\text { free survival compared } \\
\text { with standard therapy } \\
\text { (months) }\end{array}$ \\
\hline \multirow[t]{4}{*}{ EGFR } & Erlotinib & $\begin{array}{l}\text { First-generation endothelial growth factor (EGFR) } \\
\text { tyrosine kinase inhibitor (TKI) }\end{array}$ & $\begin{array}{l}9.7 \text { vs } 5.2^{10} \\
13.1 \text { vs } 4.6^{11}\end{array}$ \\
\hline & Gefitinib & First-generation EGFR TKI & $\begin{array}{l}9.2 \text { vs } 6.3^{12} \\
10.8 \text { vs } 5.4^{13}\end{array}$ \\
\hline & Afatinib & Second-generation EGFR TKI & 11.1 vs $6.9^{14}$ \\
\hline & Osimertinib & Third-generation EGFR TKI & 18.9 vs $10.2^{15 ; a}$ \\
\hline \multirow[t]{4}{*}{ ALK } & Ceritinib & First-generation ALK/ROS1/HGFR TKI & 16.6 vs $8.1^{16}$ \\
\hline & Crizotinib & First-generation ALK/ROS1/HGFR TKI & 10.9 vs $7.0^{17}$ \\
\hline & Alectinib & Second-generation ALK/ROS1/HGFR TKI & Median not reached ${ }^{18}$ \\
\hline & Brigatinib & Second-generation ALK/ROS1/HGFR TKI & 24.0 vs $11.0^{19}$ \\
\hline \multirow[t]{2}{*}{ ROS1 } & Crizotinib & First-generation ALK/ROS1/HGFR TKI & $\begin{array}{l}17.6^{20 ; b} \\
15.9^{21 ; b}\end{array}$ \\
\hline & Entrectinib & First-generation ALK/ROS1/HGFR TKI & Trials ongoing \\
\hline \multirow[t]{2}{*}{ BRAF } & Dabrafenib & BRAF V600E serine/threonine kinase inhibitor & $14.6^{22 ; c}$ \\
\hline & Trametinib & MEK $1 / 2$ Inhibitor & $14.6^{22 ; c}$ \\
\hline
\end{tabular}

\section{$B R A F$ mutations}

BRAF mutations, commonly associated with melanoma, lead to a mutated serine-threonine kinase in the MAPK kinase pathway. A BRAF mutation is the driver oncogene in $1 \%$ to $3 \%$ of cases of NSCLC. ${ }^{25}$

NSCLC BRAF mutations take multiple forms, including the classic V600E form (50\%), a G469A form (40\%), and a D594G form $(11 \%)$. Targeted therapies developed to date are primarily effective against the V600E mutation. Specific targeting of MEK1/2 mutations further downstream in the signaling pathway has also demonstrated long-term benefit and has been approved as a treatment option by the FDA.

Currently available and approved therapies for BRAF-mutant NSCLC include:

- Dabrafenib, a V600E serine/threonine kinase inhibitor
- Trametinib, a MEK 1/2 inhibitor, used in combination with dabrafenib.

Additional therapies being investigated include a combination of encorafenib with binimetinib, among others.

Common side effects of BRAF and MEK inhibitors include rash, diarrhea, and fever. A wide collection of uncommon adverse effects have been described, including systolic heart failure and retinopathy.

\section{ALK rearrangements}

ALK rearrangements lead to fusion protein products, most commonly involving echinoderm microtubule protein-like 4 (EML4). In the United States, nearly $6 \%$ of patients with NSCLC harbor an ALK rearrangement. ${ }^{23}$ The fusion in these rearrangements connects the ALK protein with exon 20 of the EML4 protein, thereby leading to constitutive activation of the ALK tyrosine kinase. Similar 
TABLE 2

\section{Definitions and descriptions of key terms}

Precision oncology -An umbrella term underscoring the personalized management of cancer patients. Precision oncology includes both the diagnostic methods required to individualize treatment of each patient's malignancy and the treatments administered based on the results of precision testing thereafter. The diagnostic methods may evaluate protein expression, cytogenetics, and mutations identified within tumor DNA. Examples of precision treatments include targeted therapies and immune checkpoint inhibitors.

Non-small cell lung cancer (NSCLC)—A broad collection of histologic findings identified in patients with lung cancer. Approximately $85 \%$ of lung cancers include NSCLC histologic findings, while $15 \%$ are small cell lung cancers. The 2 most commonly diagnosed NSCLCs are adenocarcinoma and squamous cell carcinoma. The rate of actionable mutations is far greater in patients with adenocarcinoma than in those with squamous cell carcinoma.

Driver mutation-A genetic alteration that provides a tumor cell with a fundamental growth advantage compared with normal tissue. If a targeted therapy has been discovered and validated among cancer patients harboring a specific driver mutation, the mutation may also be actionable. If a driver mutation has been studied extensively and is related to a better or worse prognosis, the mutation may be clinically relevant regardless of actionability.

Passenger mutation-A mutation discovered within tumor DNA that does not drive tumorigenesis. Patients may have both driver and passenger mutations.

Clinically relevant mutation-Mutations or alterations that may alter the course of treatment for a given patient with a specific cancer. Clinically relevant mutations may be predictive of response to targeted therapies or prognostic for standard treatment approaches.

Actionable mutation or actionable alteration-Genetic mutations or alterations that correlate with response to targeted therapies. Mutations may be within oncogenes, thereby driving tumorigenesis, or tumor suppressor genes, thereby limiting mechanisms that mitigate tumorigenesis. Mutations most frequently correspond with increased or decreased activity of critical proteins. Targeted therapies commonly exert their effects on these specific proteins. On the other hand, cytotoxic chemotherapy often drives mutations in tumor DNA, which encourages cell apoptosis.

Chromosomal rearrangement- $A$ form of genetic alteration in which 2 chromosomes are fused in abnormal combinations. The resulting proteins may drive cellular neoplastic transformation. In patients with NSCLC, rearrangements involving the $A L K$ and ROS1 genes are associated with response to targeted therapies.
Targeted treatment/therapy/agent-Drugs that specifically treat the proteins resulting from actionable genetic alterations. Within the realm of metastatic NSCLC, the most commonly prescribed targeted therapies are tyrosine kinase inhibitors (TKIs), which target the hyperactivity of the epidermal growth factor receptor.

Precision testing-Diagnostic tests conducted on resected tumor samples or tumor DNA collected and centrifuged from the blood of cancer patients that evaluate the potential response to targeted therapies. Protein expression, chromosomal rearrangements, and tumor DNA sequencing may be evaluated by precision testing.

Immunohistochemical (IHC) staining-A technique used by pathologists to visualize antigens (proteins) expressed on tumor cells. Two types of antibodies are used to indicate antigen: one antibody binds to the antigen, and another fluorescently labeled antibody binds to the antigen-antibody complex, thereby confirming the expression of a specific protein.

Fluorescence in situ hybridization (FISH)-Similar to IHC, FISH analysis uses patient tissue samples for a histologybased assay of genetic variants. However, unlike IHC, FISH probes are predicated on complementary binding that can identify specific genetic sequences of interest. Using fluorescently labeled DNA or RNA probes created to reciprocally bind targets of interest, FISH analyses are able to detect the presence of their target sequences, and thus genetic variants, within prepared tissue samples.

Tumor DNA sequencing-A broad term encompassing the various modalities to evaluate tumor DNA for mutations that may be clinically relevant. The DNA findings from a patient's tumor sample are compared with standard databases to confirm the presence of mutations. Tumor DNA sequencing may assess the DNA of certain genes, whole exomes, or the entire genome.

Next-generation sequencing (NGS) — A form of tumor DNA sequencing in which massive amplification of preselected portions of tumor DNA can be evaluated concurrently. Several complementary DNA probes are affixed to comprehensive NGS plates that allow for multiple portions of DNA to be sequenced simultaneously. The data output may be in the form of fluorescence, temperature, or current change, depending on the design of the NGS platform. Given the large volume of data generated concurrently, large-scale automated algorithms are required to process cumulative sequencing information.

Liquid biopsy or plasma genotyping - A form of NGS that is conducted on DNA from dead tumor cells identified in the blood of patients with cancer. Liquid biopsy requires the collection and separation of circulating tumor DNA using advanced centrifuge techniques. 


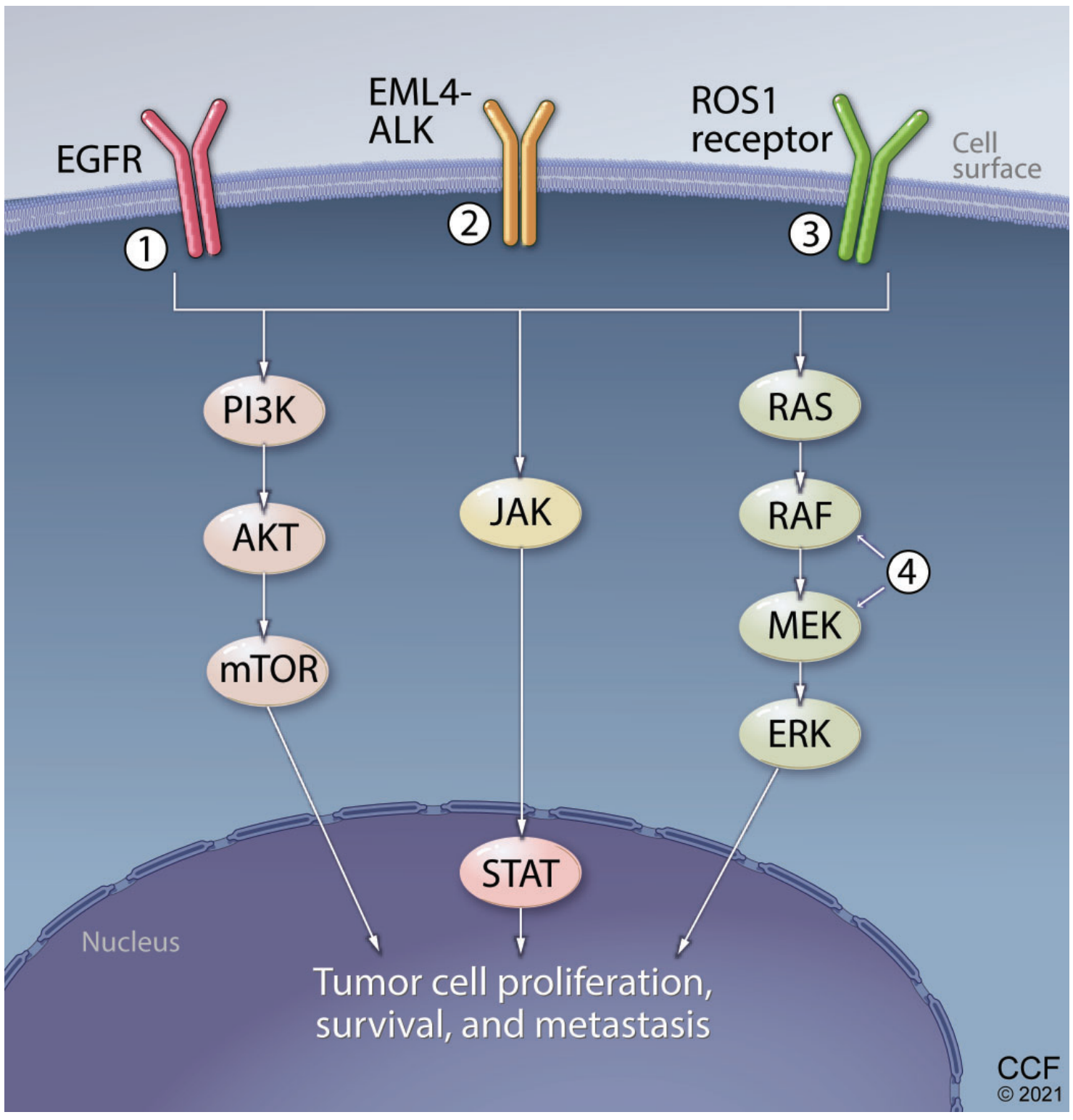

$\mathrm{AKT}=$ protein kinase $\mathrm{B} ; \mathrm{ALK}=$ anaplastic lymphoma kinase; $\mathrm{BRAF}=\mathrm{B}$-rapidly accelerated fibrosarcoma; $\mathrm{EGFR}=$ epidermal growth factor receptor; EML4 = echinoderm microtubule-associated protein-like 4; ERK = extracellular regulated kinase; JAK = Janus kinase; MEK = mitogen-activated protein kinase kinase; $\mathrm{mTOR}$ = mechanistic target of rapamycin; PI3K = phosphatidylinositol 3-kinase; $\mathrm{RAF}=$ rapidly accelerated fibrosarcoma; RAS = rat sarcoma; ROS1 = reactive oxygen species proto-oncogene 1, receptor tyrosine kinase; STAT = signal transducer and transcription

Figure 3. Pathways of proliferation. Certain key proteins that are abnormally active due to mutations and genetic rearrangements contribute to tumor cell proliferation, survival, and metastasis. Targeted therapies can block these pathways, specifically inhibitors of (1) epidermal growth factor receptor (EGFR), (2) anaplastic lymphoma kinase (ALK), (3) ROS1, (4) BRAF/MEK, and others.

to EGFR mutations, the ALK rearrangement creates a downstream transduction pathway via the AKT and ERK signaling pathways that encourages growth and discourages apoptosis.
ALK inhibitors have demonstrated excellent outcomes among patients with metastatic ALK-rearranged NSCLC.

Common adverse effects with ALK inhibitors include gastrointestinal toxicities. Brady- 
cardia, QT prolongation, and interstitial lung disease are possible.

Currently available and approved ALK inhibitors are:

- Crizotinib, a first-generation drug

- Ceritinib, a first-generation drug

- Alectinib, a second-generation drug

- Brigatinib, a second-generation drug

- Lorlatinib, a third-generation drug.

\section{ROS1 rearrangements}

Rearrangements of the receptor tyrosine kinase c-ros oncogene 1 (ROS1) on chromosome 6 lead to constitutive tyrosine kinase activity, stimulating oncogenic signals through downstream pathways. Importantly, the ROS1 rearrangements result in a mutant protein form that is structurally very similar to that seen among ALK rearrangements. That structural similarity creates cross-sensitivity and cross-reactivity with broad-target tyrosine kinase inhibitors, allowing for use of these targeted therapies in patients with ROS1 rearrangements in addition to their originally intended targets. Approximately 1\% of patients with NSCLC in the United States harbor a ROS1 rearrangement.

Currently approved therapies include:

- Crizotinib, first-generation

- Entrectinib, first-generation.

Other tyrosine kinase inhibitors in development or recommended as alternative therapies include ceritinib.

Adverse effects are drug-dependent. Targeted agents that concurrently serve as ALK inhibitors, such as crizotinib, share the aforementioned ALK-inhibitor risk profiles. On the other hand, entrectinib is part of a separate collection of drugs that are typically prescribed for patients with neurotrophic tyrosine receptor kinase (NTRK) gene fusions in other solid tumors. Patients receiving these drugs may face a separate group of adverse effects, most commonly fatigue, liver and kidney dysfunction, and myelosuppression.

\section{MET, RET, AND OTHERS}

In the summer of 2020, the FDA approved treatments for patients harboring alterations in RET (selpercatinib and pralsetinib) and MET (capmatinib). ${ }^{26-28}$

However, these alterations represent only a fraction of the spectrum of pathogenic alterations in NSCLC; many more are currently being investigated in the laboratory and through clinical research. These include alterations in KRAS, NRAS, AKT, DDR2, HER2 (ERBB2), PIK3CA, MEK1, PTEN, and FGFR. ${ }^{29,30}$

This list, and our understanding of how these alterations drive tumorigenesis in NSCLC, will continue to expand in the years to come.

\section{TESTS FOR CLINICALLY RELEVANT MUTATIONS}

Precision oncology requires equal emphasis on new drugs and identifying the patients most likely to benefit from them. Medical oncologists constantly face decisions about the best diagnostic test and timing of testing for their patients with NSCLC. A thorough understanding of the tests available is therefore critically important to the delivery of the best possible care.

The current diagnostic tests include:

- Immunohistochemical (IHC) staining

- Fluorescence in situ hybridization (FISH)

- Reverse transcriptase polymerase chain reaction (RT-PCR)

- Tissue-based next-generation sequencing (NGS).

The diagnostic accuracy, breadth of mutations, financial cost, and time required for each test vary considerably.

\section{Immunohistochemical staining}

IHC staining is a histology-based analytical tool for identifying mutational variants through specialized stains and targeted antibodies to demonstrate the presence or absence of a genetic variant within the tissue sample. It is largely used as a screening tool, given its demonstrated ability to efficiently capture identifiable variants.

Multiple studies have demonstrated sensitivity ranging from $86 \%$ to $100 \%$ and specificity of $76 \%$ to $100 \%$ for detecting ALK variants, with similar evidence for detecting EGFR mutations. ${ }^{31-35}$

The cost of IHC ranges from $\$ 33$ to $\$ 124$ and averages $\$ 73$, making it the cheapest test for mutations. ${ }^{36}$ IHC testing for ALK is FDAapproved, with FISH used for equivocal cases. For ROS1, IHC may be used in screening, but

\section{Precision} oncology requires equal emphasis on new drugs and identifying the patients most likely to benefit from them 
further FISH, PCR, or NGS testing should be used to confirm positive results and rule out false-positive results.

Because of limited sensitivity in detecting specific EGFR mutations, using IHC to determine candidacy for targeted agents against EGFR is discouraged in current guidelines for mutational testing. ${ }^{37}$

\section{Fluorescence in situ hybridization}

Similar to IHC, FISH analysis utilizes patient tissue samples for a histology-based assay of genetic variants. However, unlike IHC, FISH probes are predicated on complementary binding that can identify specific genetic sequences of interest. Using fluorescently labeled DNA or RNA probes created to reciprocally bind targets of interest, FISH analyses can detect the presence of their target sequences, and thus genetic variants, within prepared tissue samples.

FISH remains the gold standard for detecting mutant fusion protein variants and is still widely used for this purpose today. The sensitivity ranges between $90.3 \%$ and $100 \%$ and the specificity between $97.7 \%$ and $100 \%$ among patients being tested for ALK rearrangements. ${ }^{38,39}$ Cost of FISH testing averages about $\$ 300$, and turnaround processing time averages about 2 to 5 days, marginally longer than that of IHC processing. ${ }^{36}$

The most significant drawbacks of FISH testing arise from its limited scope (each test is specific for 1 genetic variant), need for fluorescent microscope workstations, and the qualitative component of its assessment (there may be some ambiguity based on the cutoff point for positive vs negative results). ${ }^{40}$

\section{Reverse transcriptase polymerase chain reaction-based methods}

RT-PCR analysis uses unique, labeled DNA probes to identify, amplify, and quantify the levels of specific genetic variants in tissue samples. It has demonstrated efficacy and accuracy as a stand-alone diagnostic tool and in comparison to IHC, FISH, and NGS. ${ }^{41} \mathrm{Ad}$ vantages: it can perform multiple simultaneous assessments, it can be done on samples other than biopsy tissue (such as blood), and it is objective-there is no subjective rating of positivity as in IHC and FISH. Its sensitivity for identifying mutational variants ranges from $88 \%$ to $100 \%$ and its specificity from $94 \%$ to $100 \% .^{39,42,43}$

While the individual costs of a single RTPCR assay are difficult to characterize owing to the variability of pricing of reagents, technical labor, and available facilities, multiple studies have demonstrated the cost-effectiveness of RT-PCR testing in comparison to histologybased diagnostic tools.

\section{Tissue-based next-generation sequencing}

By identifying the full genetic sequences of targeted areas of the genome, NGS is able to identify both documented and previously undiscovered mutational variants by similar principles of complementary nucleotide binding as RT-PCR, but at a larger scale. ${ }^{44}$ This broad applicability allows for interrogation of an ever-expanding library of driver mutations, all at once, with pinpoint accuracy.

Advances in NGS technology over the last several years have driven down overall costs while improving accuracy and ease of application, making economical feasibility a reality. NGS is now commonly used in genetic assessment in advanced NSCLC. ${ }^{45-47}$ In its earliest iterations, NGS was demonstrated to have high sensitivity and specificity values by validation studies (95\%-99\%, with positive predictive value $>99 \%) .^{48}$ More recent studies have assessed these markers of accuracy at $100 \%$ for both sensitivity and specificity, establishing NGS as the comparative technique against which other mutation identification processes can be evaluated. ${ }^{49,50}$

However, the estimated cost of targeted gene panel sequencing averages $\$ 1,609$, with significant variation depending on the size of the panel of mutational targets, preference for whole-exome sequencing $(\$ 4,459)$, or wholeexome plus RNA sequencing $(\$ 5,938) .{ }^{45}$ In addition, turnaround times for NGS studies are long, with estimates of 13 to 21 days on average in multiple studies. ${ }^{51}$

\section{Plasma genotyping}

Plasma genotyping, popularly called "liquid biopsy," a broad collection of screening tests utilizing capture and identification of circulating tumor DNA (ctDNA), has demonstrated incredible promise in its early forms. ${ }^{52-55}$ It has significant clinical potential, given its ease of implementation, low risk compared with tis- 
sue-dependent screening methods, rapid turnaround time, and ability to perform screening analysis without limitations (eg, amount of tissue collected, need for repeat biopsy). This technology may allow for detection of new actionable mutations, characterization of response to therapy, and identification of mechanisms of resistance to therapy ${ }^{56,57}$ Early assessments have demonstrated some level of agreement between ctDNA assessments and previously confirmed tissue diagnoses, with high levels of individualized variant identification by ctDNA alone.

\section{HOW HAS NEXT-GENERATION SEQUENCING ALTERED TESTING PRACTICES?}

Clinicians practicing precision medicine must carefully consider the cost-benefit analysis of this approach and plan their diagnostic and therapeutic course accordingly: What actionable information will result from testing? What testing method will provide maximal utilizable information at the lowest cost? What is the feasibility of implementing a therapy based on that information?

Clinicians can use a wide array of testing procedures that have well-documented clinical efficacy, from histology-based IHC analyses to small-scale quantitative PCR assays. Employing these tests for initial screening, especially in settings with limited access to advanced technologies or ability to follow through on the data they provide, even for a faster stepwise diagnostic approach, could allow clinical oncologists to refine their approach to diagnosis and treatment in the precision medicine era.

NGS technology provides an unparalleled view of the genetic framework of a patient's disease. It allows clinicians and researchers to identify a significant proportion of the full mutational burden of a tumor and uncover the various targets for which therapies can be used. This has created many opportunities for research and clinical investigation of this technology, opening the door for trials explor- ing the efficacy of a wide range of therapies.

Looking ahead, application of NGS technology to ctDNA isolated from simple blood samples continues to expand the landscape of precision medicine. The potential to identify and exhaustively characterize tumors with rapid, noninvasive diagnostic tools is incredibly appealing. Like NGS technology and the precision oncology movement as a whole, the inherent potential for paradigm-shifting clinical impact will continue to drive interest in this technology.

\section{FUTURE DIRECTIONS FOR RESEARCH AND CLINICAL PRACTICE}

As research advances our understanding of the molecular framework of NSCLC, clinicians must stay informed about the latest testing methods and therapies, actionable mutations, and breakthrough approaches. Research into the EGFR, BRAF, ALK, ROS1, and other alterations driving disease has unlocked treatments that have changed the course of disease in countless patients. The use of precision medicine in NSCLC will benefit patients for years to come.

Future discussions of the research and therapies surrounding NSCLC will necessarily focus on:

- Discovery of new driver mutations

- New therapies that target these currently unidentified mutations

- Advances in currently developed therapies

- Results of clinical trials and bench research currently in progress

- Expansion and streamlining of the testing procedures used for variant identification (ie, genomic sequencing).

\section{DISCLOSURES}

Dr. Clarke reports financial relationships (consulting, independent contracting, or research) with AbbVie Pharmaceuticals, Adaptimmune, Array BioPharma, AstraZeneca, Bristol Myers Squibb, Genentech, Grid Therapeutics, Guardant Health, Merck, Moderna, NGM Biopharmaceuticals, Pfizer, and Spectrum Pharmaceuticals.

All other authors report no relevant financial relationships which, in the context of their contributions, could be perceived as a potential conflict of interest. 


\section{REFERENCES}

1. Maemondo M, Inoue A, Kobayashi K, et al. Gefitinib or chemotherapy for non-small-cell lung cancer with mutated EGFR. N Engl J Med 2010; 362(25):2380-2388. doi:10.1056/NEJMoa0909530

2. Rosell R, Carcereny E, Gervais R, et al. Erlotinib versus standard chemotherapy as first-line treatment for European patients with advanced EGFR mutation-positive non-small-cell lung cancer (EURTAC): a multicentre, open-label, randomised phase 3 trial. Lancet Oncol 2012; 13(3):239-246. doi:10.1016/S1470-2045(11)70393-X

3. Soria J-C, Tan DSW, Chiari R, et al. First-line ceritinib versus platinum-based chemotherapy in advanced ALK-rearranged nonsmall-cell lung cancer (ASCEND-4): a randomised, open-label, phase 3 study. Lancet 2017; 389(10072):917-929. doi:10.1016/S0140-6736(17)30123-X

4. Ramalingam S, Dinan MA, Crawford J. Survival comparison in patients with stage IV lung cancer in academic versus community centers in the United States. J Thorac Oncol 2018; 13(12):1842-1850. doi:10.1016/j.jtho.2018.09.007

5. Aguiar PN Jr, Haaland B, Park W, San Tan P, Del Giglio A, de Lima Lopes G Jr. Cost-effectiveness of osimertinib in the first-line treatment of patients with EGFR-mutated advanced non-small cell lung cancer. JAMA Oncol 2018; 4(8):1080-1084. doi:10.1001/jamaoncol.2018.1395

6. Holleman MS, AI MJ, Zaim R, Groen HJM, Uyl-de Groot CA. Costeffectiveness analysis of the first-line EGFR-TKIs in patients with non-small cell lung cancer harbouring EGFR mutations. Eur J Health Econ 2020; 21(1):153-164. doi:10.1007/s10198-019-01117-3

7. Sivignon M, Monnier R, Tehard B, Roze S. Cost-effectiveness of alectinib compared to crizotinib for the treatment of first-line ALK+ advanced non-small-cell lung cancer in France. PLoS One 2020; 15(1):e0226196. doi:10.1371/journal.pone.0226196

8. Chan BA, Hughes BG. Targeted therapy for non-small cell lung cancer: current standards and the promise of the future. Transl Lung Cancer Res 2015; 4(1):36-54. doi:10.3978/j.issn.2218-6751.2014.05.01

9. Merid SK, Goranskaya D, Alexeyenko A. Distinguishing between driver and passenger mutations in individual cancer genomes by network enrichment analysis. BMC Bioinformatics 2014; 15(1):308 doi:10.1186/1471-2105-15-308

10. Rosell R, Carcereny E, Gervais R, et al. Erlotinib versus standard chemotherapy as first-line treatment for European patients with advanced EGFR mutation-positive non-small-cell lung cancer (EURTAC): a multicentre, open-label, randomised phase 3 trial. Lancet Onco 2012; 13(3):239-246. doi:10.1016/S1470-2045(11)70393-X

11. Zhou C, Wu Y-L, Chen G, et al. Erlotinib versus chemotherapy as first-line treatment for patients with advanced EGFR mutationpositive non-small-cell lung cancer (OPTIMAL, CTONG-0802): a multicentre, open-label, randomised, phase 3 study. Lancet Onco 2011; 12(8):735-742. doi:10.1016/S1470-2045(11)70184-X

12. Mitsudomi T, Morita S, Yatabe $Y$, et al. Gefitinib versus cisplatin plus docetaxel in patients with non-small-cell lung cancer harbouring mutations of the epidermal growth factor receptor (WJTOG3405): an open label, randomised phase 3 trial. Lancet Oncol 2010; 11(2):121-128. doi:10.1016/S1470-2045(09)70364-X

13. Maemondo M, Inoue A, Kobayashi K, et al. Gefitinib or chemotherapy for non-small-cell lung cancer with mutated EGFR. N Engl J Med 2010; 362(25):2380-2388. doi:10.1056/NEJMoa0909530

14. Sequist LV, Yang JC-H, Yamamoto N, et al. Phase III study of afatinib or cisplatin plus pemetrexed in patients with metastatic lung adenocarcinoma with EGFR mutations. J Clin Oncol 2013; 31(27):33273334. doi:10.1200/JCO.2012.44.2806

15. Soria J-C, Ohe $\mathrm{Y}$, Vansteenkiste J. Osimertinib in untreated EGFRmutated advanced non-small-cell lung cancer. N Engl J Med 2018; 378(2):113-125. doi:10.1056/NEJMoa1713137

16. Soria J-C, Tan DSW, Chiari R, et al. First-line ceritinib versus platinum-based chemotherapy in advanced ALK-rearranged nonsmall-cell lung cancer (ASCEND-4): a randomised, open-label, phase 3 study. Lancet 2017; 389(10072):917-929. doi:10.1016/S0140-6736(17)30123-X
17. Solomon BJ, Mok T, Kim D-W, et al. First-line crizotinib versus chemotherapy in ALK-positive lung cancer. N Engl J Med 2014 371(23):2167-2177. doi:10.1056/NEJMoa1408440

18. Peters S, Camidge DR, Shaw AT, ALEX Trial Investigators. Alectinib versus crizotinib in untreated ALK-positive non-small-cell lung cancer. N Engl J Med 2017; 377(9):829-838. doi:10.1056/NEJMoa1704795

19. Camidge DR, Kim HR, Ahn M-J, et al. Brigatinib versus crizotinib in advanced ALK inhibitor-naive ALK-positive non-small cell lung cancer: second interim analysis of the phase III ALTA-1L trial. J Clin Oncol 2020; 38(31):3592-3603. doi:10.1200/JCO.20.00505

20. Shaw AT, Ou S-H, Bang Y-J, et al. Crizotinib in ROS1-rearranged non-small-cell lung cancer. N Engl J Med 2014; 371(21):1963-1971. doi:10.1056/NEJMoa1406766

21. Wu Y-L, Yang JC-H, Kim D-W, et al. Phase II study of crizotinib in East Asian patients with ROS1-positive advanced non-small-cell lung cancer. J Clin Oncol 2018; 36(140:1405-1411. doi:10.1200/JCO.2017.75.5587

22. Planchard D, Smit EF, Groen HJM, et al. Dabrafenib plus trametinib in patients with previously untreated BRAF V600E-mutant metastatic non-small-cell lung cancer: an open-label, phase 2 trial. Lancet Oncol 2017; 18(10):1307-1316. doi:10.1016/S1470-2045(17)30679-4

23. Suh JH, Johnson A, Albacker L, et al. Comprehensive genomic profiling facilitates implementation of the national comprehensive cancer network guidelines for lung cancer biomarker testing and identifies patients who may benefit from enrollment in mechanism-driven clinical trials. Oncologist 2016; 21(6):684-691. doi:10.1634/theoncologist.2016-0030

24. Griffin R, Ramirez RA. Molecular targets in non-small cell lung cancer. Ochsner J 2017; 17(4):388-392. pmid:29230123

25. Baik CS, Myall NJ, Wakelee HA. Targeting BRAF-mutant non-smal cell lung cancer: from molecular profiling to rationally designed therapy. Oncologist 2017; 22(7):786-796. doi:10.1634/theoncologist.2016-0458

26. US Food and Drug Administration. FDA approves selpercatinib for lung and thyroid cancers with RET gene mutations or fusions. May 11, 2020. Accessed January 5, 2021. https://www.fda.gov/drugs/ drug-approvals-and-databases/fda-approves-selpercatinib-lung-andthyroid-cancers-ret-gene-mutations-or-fusions

27. US Food and Drug Administration. FDA approves pralsetinib for lung cancer with RET gene fusions. September 8, 2020. Accessed January 5, 2021. https://www.fda.gov/drugs/resources-informationapproved-drugs/fda-approves-pralsetinib-lung-cancer-ret-genefusions

28. US Food and Drug Administration. FDA approves first targeted therapy to treat aggressive form of lung cancer. May 6, 2020. Accessed January 5, 2021. https://www.fda.gov/news-events/press-announcements/fda-approves-first-targeted-therapy-treat-aggressiveform-lung-cancer

29. Desai A, Menon SP, Dy GK. Alterations in genes other than EGFR/ ALK/ROS1 in non-small cell lung cancer: trials and treatment options. Cancer Biol Med 2016; 13(1):77-86. doi:10.28092/j.issn.2095-3941.2016.0008

30. Cancer Genome Atlas Research Network. Comprehensive molecular profiling of lung adenocarcinoma. Nature 2014; 511(7511):543-550. doi:10.1038/nature13385

31. Niu X, Chuang JC, Berry GJ, Wakelee HA. Anaplastic lymphoma kinase testing: IHC vs. FISH vs. NGS. Curr Treat Options Oncol 2017; 18(12):71. doi:10.1007/s11864-017-0513-x

32. Sholl LM, Weremowicz S, Gray SW, et al. Combined use of ALK immunohistochemistry and FISH for optimal detection of ALK-rearranged lung adenocarcinomas. J Thorac Oncol 2013; 8(3):322-328. doi:10.1097/JTO.0b013e31827db604

33. Cruz-Rico G, Avilés-Salas A, Segura-González M, et al. Diagnosis of EML4-ALK translocation with FISH, immunohistochemistry, and realtime polymerase chain reaction in patients with non-small cell lung cancer. Am J Clin Oncol 2017; 40(6):631-638. doi:10.1097/COC.0000000000000213

34. Hitij NT, Kern I, Sadikov A, et al. Immunohistochemistry for EGFR mutation detection in non-small-cell lung cancer. Clin Lung Cancer 


\section{LEE AND COLLEAGUES}

2017; 18(3):e187-e196. doi:10.1016/j.cllc.2016.11.021

35. Houang $\mathbf{M}$, Toon CW, Clarkson A, et al. Reflex ALK immunohistochemistry is feasible and highly specific for ALK gene rearrangements in lung cancer. Pathology 2014; 46(5):383-388. doi:10.1097/PAT.0000000000000114

36. Doshi S, Ray D, Stein K, et al. Economic analysis of alternative strategies for detection of ALK rearrangements in non small cell lung cancer. Diagnostics (Basel, Switzerland) 2016; 6(1):4. doi:10.3390/diagnostics6010004

37. Lindeman NI, Cagle PT, Aisner DL, et al. Updated molecular testing guideline for the selection of lung cancer patients for treatment with targeted tyrosine kinase inhibitors: guideline from the College of American Pathologists, the International Association for the Study of Lung Cancer, and the Association for Molecular Pathology. Arch Pathol Lab Med 2018; 142(3):321-346 doi:10.5858/arpa.2017-0388-CP

38. Pekar-Zlotin M, Hirsch FR, Soussan-Gutman L, et al. Fluorescence in situ hybridization, immunohistochemistry, and next-generation sequencing for detection of EML4-ALK rearrangement in lung cancer. Oncologist 2015; 20(3):316-322.

doi:10.1634/theoncologist.2014-0389

39. Demidova I, Barinov A, Savelov N, et al. Immunohistochemistry, fluorescence in situ hybridization, and reverse transcription-polymerase chain reaction for the detection of anaplastic lymphoma kinase gene rearrangements in patients with non-small cell lung cancer: potential advantages and methodologic pitfalls. Arch Patho Lab Med 2014; 138(6):794-802. doi:10.5858/arpa.2012-0762-OA

40. Ma D, Wang Z, Yang L, et al. Responses to crizotinib in patients with ALK-positive lung adenocarcinoma who tested immunohistochemistry (IHC)-positive and fluorescence in situ hybridization (FISH)negative. Oncotarget 2016; 7(39):64410-64420. doi: 10.18632/oncotarget. 10560

41. Wang Y, Zhang J, Gao G, et al. EML4-ALK fusion detected by RT-PCR confers similar response to crizotinib as detected by FISH in patients with advanced non-small-cell lung cancer. J Thorac Oncol 2015; 10(11):1546-1552. doi:10.1097/JTO.0000000000000668

42. Shin S, Kim J, Kim Y, Cho SM, Lee KA. Assessment of real-time PCR method for detection of EGFR mutation using both supernatant and cell pellet of malignant pleural effusion samples from non-small-cell lung cancer patients. Clin Chem Lab Med 2017; 55(12):1962-1969. doi:10.1515/cclm-2016-0851

43. Hout DR, Schweitzer BL, Lawrence K, et al. Performance of a RT-PCR assay in comparison to FISH and immunohistochemistry for the detection of ALK in non-small cell lung cancer. Cancers 2017; 9(8):99. doi:10.3390/cancers9080099

44. Hou H, Yang X, Zhang J, et al. Discovery of targetable genetic alterations in advanced non-small cell lung cancer using a nextgeneration sequencing-based circulating tumor DNA assay. Sci Rep 2017; 7(1):14605. doi:10.1038/s41598-017-14962-0

45. Tan O, Shrestha R, Cunich M, Schofield DJ. Application of nextgeneration sequencing to improve cancer management: a review of the clinical effectiveness and cost-effectiveness. Clin Genet 2018; 93(3):533-544. doi:10.1111/cge.13199

46. Dalal AA, Guerin A, Mutebi A, Culver KW. Economic analysis of BRAF gene mutation testing in real world practice using claims data: costs of single gene versus panel tests in patients with lung cancer. J Med Econ 2018; 21(7):649-655. doi:10.1080/13696998.2018.1450261

47. Sabatini LM, Mathews C, Ptak D, et al. Genomic sequencing procedure microcosting analysis and health economic cost-impact analysis: a report of the Association for Molecular Pathology. J Mol Diagn 2016; 18(3):319-328. doi:10.1016/j.jmoldx.2015.11.010

48. Frampton GM, Fichtenholtz A, Otto GA, et al. Development and validation of a clinical cancer genomic profiling test based on massively parallel DNA sequencing. Nature Biotech 2013; 31(11):1023-1031. doi:10.1038/nbt.2696

49. Pfarr N, Stenzinger A, Penzel R, et al. High-throughput diagnostic profiling of clinically actionable gene fusions in lung cancer. Genes Chromosomes Cancer 2016; 55(1):30-44. doi:10.1002/gcc.22297

50. Ding S, Liu N, Zhao H, Jiang G, Zhang X, Wang E. Significance and evaluation of anaplastic lymphoma kinase by immunohistochemistry in non-small cell lung cancer. Tumour Biol 2016; 37(8):10917-10922. doi:10.1007/s13277-016-4946-9

51. Hagemann IS, Devarakonda S, Lockwood CM, et al. Clinical nextgeneration sequencing in patients with non-small cell lung cancer. Cancer 2015; 121(4):631-639. doi:10.1002/cncr.29089

52. Kim E, Feldman R, Wistuba, II. Update on EGFR mutational testing and the potential of noninvasive liquid biopsy in non-small-cell lung cancer. Clin Lung Cancer 2018; 19(2):105-114. doi:10.1016/j.cllc.2017.08.001

53. Thompson JC, Yee SS, Troxel AB, et al. Detection of therapeutically targetable driver and resistance mutations in lung cancer patients by next-generation sequencing of cell-free circulating tumor DNA. Clin Cancer Res 2016; 22(23):5772-5782. doi:10.1158/1078-0432.CCR-16-1231

54. Liu L, Liu H, Shao D, et al. Development and clinical validation of a circulating tumor DNA test for the identification of clinically actionable mutations in nonsmall cell lung cancer. Genes Chromosomes Cancer 2018; 57(4):211-220. doi:10.1002/gcc.22522

55. Mayo-de-Las-Casas C, Jordana-Ariza N, Garzon-lbanez M, et al. Large scale, prospective screening of EGFR mutations in the blood of advanced NSCLC patients to guide treatment decisions. Ann Oncol 2017; 28(9):2248-2255. doi:10.1093/annonc/mdx288

56. McCoach CE, Blakely CM, Banks KC, et al. Clinical utility of cell-free DNA for the detection of ALK fusions and genomic mechanisms of ALK inhibitor resistance in non-small cell lung cancer. Clin Cancer Res 2018; 24(12):2758-2770. doi:10.1158/1078-0432.CCR-17-2588

57. Duffy MJ. Circulating tumour DNA as a cancer biomarker. Ann Clin Biochem 2019; 56(1):42-48. doi:10.1177/0004563218798401

Address: Vishal Vashistha, MD, Raymond G. Murphy Department of Veterans Affairs Medical Center, 1501 San Pedro Drive, Southeast, Albuquerque, NM 87108-5153; vashistha87@gmail.com 